\title{
State of XR research in architecture with focus on professional practice - a systematic literature review
}

\section{Adeline Stals \& Luisa Caldas}

To cite this article: Adeline Stals \& Luisa Caldas (2020): State of XR research in architecture with focus on professional practice - a systematic literature review, Architectural Science Review, DOI: 10.1080/00038628.2020.1838258

To link to this article: https://doi.org/10.1080/00038628.2020.1838258

曲 Published online: 20 Nov 2020.

Submit your article to this journal $\widetilde{ }$

III Article views: 63

Q View related articles $₫$

View Crossmark data $₫$ 


\title{
State of XR research in architecture with focus on professional practice - a systematic literature review
}

\author{
Adeline Stals $(1)$ and Luisa Caldas \\ College of Environmental Design, University of California, Berkeley, CA, USA
}

\begin{abstract}
Immersive technologies are not only gaining popularity in various fields but are also heralded as the obvious next step in architectural practice. Now that almost five years have passed since the release of more accurate and affordable headsets, a review focusing on immersive technology applications in the architectural field is needed to reflect the current fields investigated. This systematic literature review discusses the sample used in the 201 selected studies about immersive technologies published from 2015 to 2019 . The study identifies gaps in the current literature. The results highlight that professional architects are almost never queried in searches conducted over the past five years in the selected database. It unveils the necessity to take into consideration the context of studies in order to develop tools truly dedicated to the real practices of professional architects. This paper constitutes a reference for further researches by facilitating their contextualization within the research landscape.
\end{abstract}

\section{ARTICLE HISTORY}

Received 13 April 2020

Accepted 14 October 2020

\section{KEYWORDS}

Literature review; professional practice; virtual reality; augmented reality; mixed reality; immersive technology

\section{Introduction}

Immersive technologies have been improved in terms of variety, display resolution, sensors accuracy and at the same time have become more affordable. Due to the successful implementation of these technologies across various sectors, including the aviation, medicine, military for example (Wang and Dunston 2007; Jeelani, Han, and Albert 2017; Krakhofer and Kaf$\tan 2015)$, researchers have started to analyse the interest for the AEC industry (Greenwood et al. 2008; Cheng, Chen, and Chen 2020). The interest for onsite applications (Billinghurst and Henrysson 2009), safety (Park et al. 2015; Moore and Gheisari 2019), facility management (Shi et al. 2016) have notably been demonstrated.

New and more efficient tools are constantly being proposed to architects. This evolution amplifies a technological wave that sometimes stimulates them, sometimes overwhelms them, depending essentially on their digital culture and the context (socio-economic, structural, organizational) in which they work on a daily basis (Bourbonnais 2014; Carpo 2017). This time, the 2013 release of the first Oculus Rift, followed by the more popular 2014 release of the Google Cardboard (Bell 2016), did not pass unnoticed by the architectural industry. These releases have contributed to the growing popularity of immersive technology in the field and even announced it as the obvious next step in architectural practice.

Beyond these different approaches and the development of new prototypes and methodologies, the potential implementation of these technologies in professional practice and therefore in the daily work of architects requires particular attention.
Already ten years ago, Moloney opened up this question: 'Perhaps more importantly for design practice, how might these technologies be implemented in a studio design environment?' (Moloney 2009, 147).

Despite the current attention paid to immersive technology, there is a lack of overview about the types of recent studies that have been conducted, the conditions under which studies have been undertaken, the challenges they address. The goal of this paper is to review contemporary researches to explore how immersive technologies are developed and tested in the architectural design process. The review, therefore, identifies gaps that will help contextualize future research. Such a contribution can benefit both industry and academia to understand the development requirements of immersive technologies for their successful long-term integration into architectural practice.

In this sense, this work proposes an investigation about the sample generally used in research about immersive technologies in architecture. To support our analysis, we present a systematic literature review on immersive technologies comprising 5 years of studies (2015-2019) from two types of databases related to the architectural field. We determine eleven categories and classified the selected papers accordingly. Afterward, we analyse and discuss the literature review in order to offer our remarks about the importance of considering the sample used in the methodology and discuss its influence on the results.

Before developing the core of the research, the next section introduces the current context of immersive technologies and highlights the research question. 


\section{The implementation of immersive technologies in architecture}

Architectural design tools have evolved over the history of the discipline, leading to the progressive transformation of architectural design practices (Schnabel 2004; Abdelhameed 2013). While sketches and scale models are still up to date, they have been accompanied by 2D drawing and 3D modelling software. Nowadays, a new technological wave is underway mainly through the development of Virtual Reality (VR) and Augmented Reality (AR) devices.

While the development of VR took his start in 1960 with the first head-mounted display invented by Morton Heilig, followed by the Ultimate display by Ivan Sutherland in 1965, the interest for immersive technologies took off in the 90 s. The evolution of representations in architecture until VR and the evolution of first devices are well described by Bertol (1996). Since then, two peaks of renewed interest in these technologies have occurred, one at the beginning of the twenty-first century and, more recently, since 2015, when the commercialization of affordable headsets has made the opportunity to benefit from immersive technologies more accessible (Heim 2017; Miltiadis 2015).

These evolutions are referred to the terms of VR (Virtual reality), AR (Augmented Reality), and MR (Mixed Reality), generally bring together under the term XR for Extended Reality. However, their differences are not always clearly defined (Hanaoka et al. 2018; Moore and Gheisari 2019; Greenwood et al. 2008). In order to better understand the distinction, we refer on definitions formulated by Milgram and his colleagues (Milgram and Kishino 1994). We, therefore, group together under the term VR, technologies in which the user is completely immersed in a fully modelled world. AR is defined as an environment in which digital representations (e.g. texts, images, videos, virtual objects) are superimposed on the physical world and 'augment' it. MR is defined as a combination in which the virtual and the real world are presented together. However, the concept of MR has evolved during the past two decades. The original concept of MR proposed by Milgram focused solely on graphics, whereas MR nowadays integrates different methods of human-computer interaction, such as environmental input, sound or gestures (Cheng, Chen, and Chen 2020). This evolution leads sometimes to an ambiguous differentiation between AR and MR. Cheng and his colleagues refer to the HoloLens released by Microsoft to exemplify this ambiguity. The device is considered an MR device, although its function is to superimpose virtual information on the real world, which can be classified as AR according to Milgram's definition. In another paper describing immersive devices, the HoloLens is this time considered as a VR devices (Spaeth and Khali 2018).

Our focus is not on a technical point-of-view but rather on the relevance of implementing new types of representation and communication through immersive technologies in architecture. Therefore, we do not deepen the explanations of these three terms and we consider in this paper all the immersive technologies developed for the architectural field. The term XR technologies is used to describe the spectrum between VR, AR and MR technologies.

These various immersive headsets offer different ways to allow stakeholders to immerse themselves in 3D representations of the design project. However, to interact and make changes in these environments, some adjustments to workflows or even the development of new ones are required (Sheldon et al. 2019). In that regard, numerous researches report the growing interest of these immersive tools in view of the new perspectives they open up in terms of representation (O'Hare et al. 2018; Schnabel et al. 2001; Caldas and Keshavarzi 2019), collaboration (Dorta et al. 2019), non-expert users involvement (Kwiecinski, Markusiewicz, and Pasternak 2017) to name but a few of the ongoing challenges.

Researches show a variety of possible uses and developments to accompany designers in their architectural design process. In parallel with the development of advanced devices, their implementation in architecture firms needs attention.

We formulate our research question in this sense: Does research on XR technology take into account feedback from professional architects? The next section is dedicated to the methodology applied to address the research question of this paper.

\section{Methodology}

In order to answer the research question raised and identify participants' profiles in recent studies about XR technologies, we have established a strict protocol for the search and appraisal of the literature. The choice for a systematic literature review allows to deal with the large number of sources, adopt a methodology that is replicable, transparent, objective, and rigorous (Boell and Cecez-Kecmanovic 2015; Snyder 2019).

Our dataset was built using a three-step process: (1) literature search, (2) systematic selection, and (3) classification.

We have firstly conducted an exploratory research in order to define the architectural reference databases. In the second step, we have established two filters, a timeframe and some keywords, to constrain the search results in databases. Finally, authors have defined categories to organize the papers selected.

\section{Literature search}

In order to find the appropriate literature, the review is based on the combination of two sources of publications dedicated to architectural content.

The first one is the Cumincad (Cumulative Index of Computer Aided Architectural Design) database which records publications from conferences exclusively about digital in architecture. This database records publications from the conferences ACADIA, CAADFutures, CAADRIA, eCAADe, SIGRADI. The number of publications selected $(n=181)$ shows the relevance of using this database as a starting point (Table 1).

Additionally to Cumincad, the second part of the corpus is based on journals publications. Journals have been selected considering their ranking on Scimago (consulted on 9 December 2019). We have considered journals in the predefined subject category 'architecture'. This topic covers journals about engineering (structures, geotechnics, energy simulation, etc.), urban planning, architectural heritage, without a specific focus on the architectural design process itself. Therefore, among the journals in Quartile 1 for 2018 (last updated when consulted), we 
Table 1. Selected publications per database and their representativeness (in percent).

\begin{tabular}{lcc}
\hline Name of database & $\begin{array}{c}\text { Number of } \\
\text { selected } \\
\text { publications }\end{array}$ & $\begin{array}{c}\text { Percentage } \\
\text { representativeness }\end{array}$ \\
\hline Cumincad & 181 & $90.05 \%$ \\
Design Studies & 2 & \\
CoDesign & 1 & \\
Architectural Science Review & 1 & \\
Frontiers of Architectural Research & 1 & \\
Archnet-IJAR & 10 & \\
IJAC & 5 & $\mathbf{1 0 0 \%}$ \\
Total & $\mathbf{2 0 1}$ & \\
\hline
\end{tabular}

have retained those that are more related to the architectural design process: Design Studies, CoDesign, Architectural Science Review, Frontiers of Architectural Research and Archnet-IJAR. Beside those journals, IJAC (International Journal of Architectural Computing), which is not classified in the 'architecture' category on Scimago, is selected by authors for its adequacy with architecture and digital.

\section{Systematic selection}

While studies generally focus on a specific type of immersive device, our objective is to consider a wide diversity of immersive technologies. The review is based on this consideration to reflect any investigation of immersive technologies into

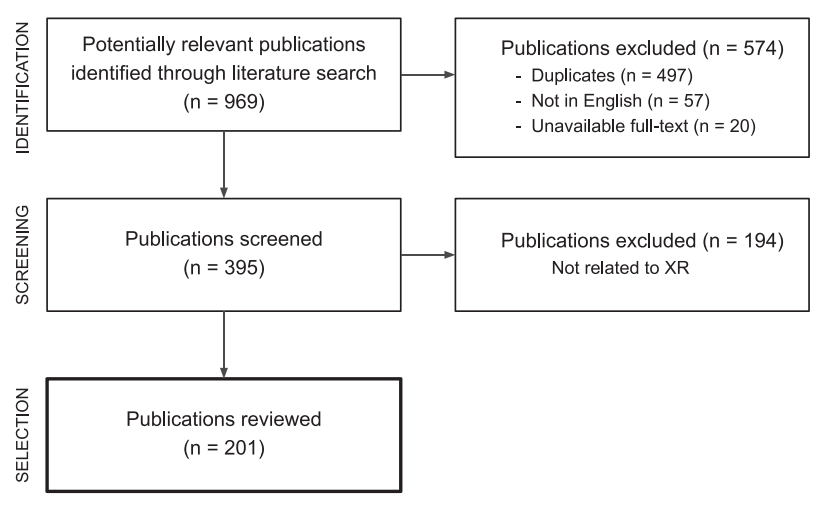

Figure 1. Systematic selection of the papers constituting our dataset. the professional architectural practice and related in scientific publications.

Therefore, for all requests, the search terms used were 'virtual reality', 'augmented reality' and 'mixed reality' in the title and/or keywords. These requests have been done through the website of each database.

After the keywords, the next filter applied restricts the publication date. The review takes into account articles published between 2015 and 2019 (included). This limited timeframe is defined considering the fast evolution of these technologies in recent years and corresponds to the third wave of immersive technologies that has made its implementation both viable and worthwhile (Heim 2017; Miltiadis 2015).

Therefore, out of the 969 papers identified through Cumincad and six architectural journals, papers occurring in two categories were removed once. We then screened the papers to select the ones meeting our inclusion criteria: full-text accessible, written in English, in the scope of at least one immersive technology (Figure 1). Accordingly, the systematic review covers 201 contemporary publications (full data are available on demand to authors).

\section{Literature classification}

The literature review is framed by a systematic examination of every article. In order to classify the selected papers, we went through each one and looked at the methodology used and more precisely at the sample of participants when available. We have defined eleven categories corresponding to different methods and samples (see Figure 2). Each paper has then been attached to one of the eleven categories. This section clarifies the meaning of each one.

In order to answer our research question on the consideration of architects in VR research, we have based our segmentation on participant' profiles. To provide an even more detailed overview of the types of research conducted and the objective pursued, we have established three categories which are named 'prototype', 'experiment' and 'theoretical approach'. We differentiate the two first based on the fact that the prototype includes the development phase of a method, an interface or the interoperability between two existing software for example. The experiment category refers more to the analysis of a situation, collecting feedback
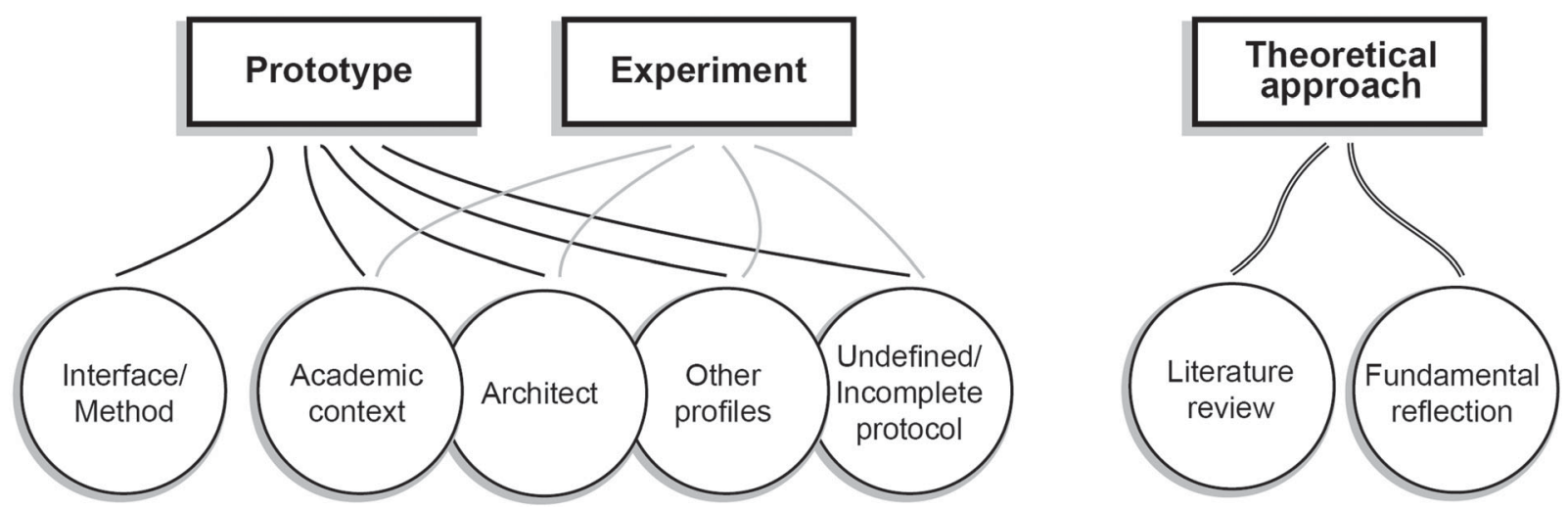

Figure 2. Relations between the three categories and the seven subcategories defined to classify each paper. 
on a technology already developed and tested in a defined environment.

For each of these two categories, we have defined four subcategories based on the participant profiles. The segmentation on participant profiles was derived from an analysis by Rogers and Schnabel (2018). They argue that findings are not necessarily transposable from one context to another and highlight in particular the difference between findings in the professional architectural practice and the educational environment. Two other categories defined by authors complete this panel to reflect the diversity of profiles that emerge from the selected articles. Therefore, we have established the following subcategories: academic context, architects, other profiles, undefined or incomplete protocol.

As a prototype can be tested or only developed and described, we have established one more subcategory, namely 'interface, method'. This subcategory 'interface, method' means that researchers explain the development of the project from a technological and technical point of view and assume that no test phase has been carried out at this stage. The research developed by Miltiadis (2015) exemplify this category. He has developed a wireless, multi-user and easily deployable augmented reality application that offers full body immersion through body, head and hand tracking.

We describe and exemplify the four common subcategories between the 'prototype' and 'experiment' categories:

- The academic context is defined by studies for which participants are students. An example is the development of a hybrid augmented reality platform to evaluate the possibilities and limitations of the fabrication, in real time and as part of the design (Poustinchi 2018). The researcher has tested the interface with volunteer students at Kent State University, College of Architecture and Environmental Design, to use the system during the early stages of their own design project;

- Some studies collect feedback from professional architects. An example of prototype tested by architects has been made by Leman and his colleagues (Figen Gul and Halici 2016) to understand the key elements of the interaction of architects with the developed interface;

- Architects are not the only actors involved in an architectural project. Therefore, some researchers focus on enhancing collaboration and integrating other profiles such as end-users. The case of citizens can be illustrated by the study conducted by Farinea, Markopoulou, and Sollazzo (2017) which focuses on fostering their participation in an urban design process;

- By undefined or incomplete protocol, we mean either that the protocol is not explained and we can not know the methodology used, or that it is incomplete in the sense that the participant profiles are not enough described. Information are missing such as the background of participants involved in the research, their affinity with the technology being tested, etc. An example considered as an undefined or incomplete protocol is the recent study by Moloney et al. (2019). They have developed an immersive VR environment and an AR application to compare their relevance for pre-occupancy evaluation at the early stages of architectural design. The prototypes are implemented for the simulation of a typical commercial office environment. Researchers have undertaken trials with people they refer to as users. This information is not complete enough to understand their area of expertise, whether or not they have different profiles, for example.

Finally, beside the 'prototype' and 'experiment' categories, we have defined a third main category under the name of 'theoretical approach'. This category encompasses researches based on a more fundamental reflection. We have identified literature reviews or considerations such as formulated by Lescop, Suner, and De Nantes (2018) under the question: 'do digital tools renew the design paradigms, or are we only involved in the evolution of practices through the integration of other means?'. These authors base their purpose on fifteen years of pedagogy going from digital drawing to virtual reality learning, without losing sight that the subject of students' work is the project and not the tool.

Since some statements have been made about the impacts of immersive environments in architecture, this categorization based on participant profiles supports our goal of identifying the context from which these findings are drawn. It then makes it possible to question more specifically how professional architects are taken into account in scientific architectural research on immersive environments.

The categories being explained, we present and discuss the results of this literature review in the following section and present an up-to-date overview of XR research for architecture.

\section{Results}

The 201 studies identified through the literature selection process are first presented in terms of distribution such as year, sources of publication or authorship. We then present the results of the sample classification.

\section{Publications distribution}

Papers were selected from two different types of databases which are CuminCad, referencing conference publications, and six architectural journals. Table 1 lists the databases used and the number of papers selected for each of them. The distribution of publications indicates that most VR-AR-MR researches have been published in conferences $(90.05 \%)$ rather than in journals (9.95\%) over the last five years. Among the journals, ArchnetIJAR has the highest number of publications with 10 papers. On the contrary, Architectural Science Review is one of the three journals selected with a single publication in the field of XR technologies between 2015 and 2019. For ASR journal, we have to go back to 1998 to find a paper on the subject. We can therefore consider XR technologies as an untapped field in ASR.

These 200 publications were written by a fairly wide variety of authors. Four names stand out with at least ten publications. Tomohiro Fukuda has published 17 papers which are almost all co-written with Nobuyoshi Yabuki who is the second most frequent author (14 papers). Both of them are from the Osaka University, Japan and have developed a focus on urban scale. Marc Aurel Schnabel counts 11 publications, co-written with Tane Moleta (10 papers). They are from Victoria University of Wellington, New Zeeland. The representation of these authors constitutes $13.9 \%$ of the selected publications of this review. 


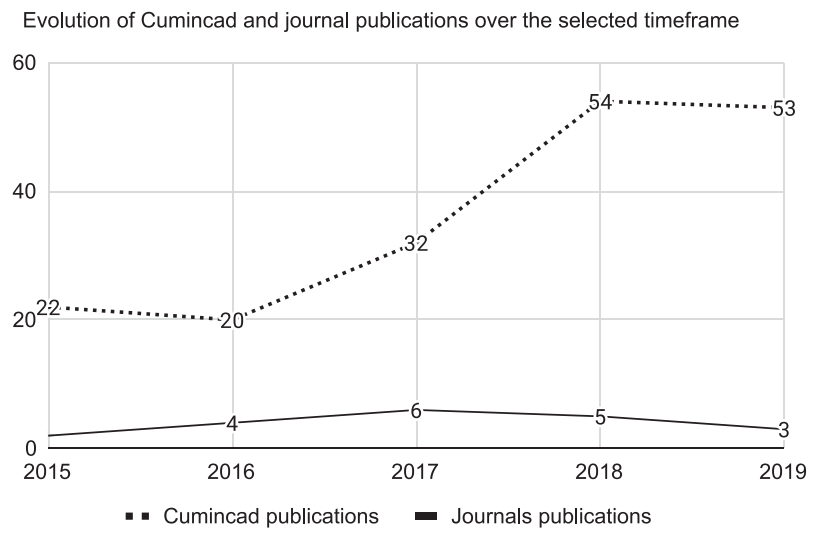

Figure 3. Number of publications in Cumincad database and architectural journals selected between 2015 and 2019.

We then organize the publications within their corresponding year range. Research publication is globally increasing over the period range from 2015 to 2019 (Figure 3). While we can observe a significant increase between 2016 and 2018 for conference publications, the number of publications in journals is quite stable with a maximum in 2017. We discuss these trends in the Discussion section.

\section{Related research samples}

The selected publications were then classified according to the methodology applied and the sample of participants who took part in the study. Table 2 shows the percentage of representation of each category.

The first observation is that the development of prototype represents more than half of the selected publications (53.73\%). The second prevailing category is experiment (35.32\%), followed by the theoretical approach (10.95\%).

We can then take a closer look at the sample of participants. In the prototype category, $34.33 \%$ of articles described a new interface or method while $19.40 \%$ of papers also reveal the first results of a user test. In this fifth of articles, the researchers rely more on the academic context $(7.96 \%)$, followed by incomplete protocol $(5.47 \%)$, then other profiles (3.98\%) and finally architects (1.99\%).

For the experiment category, the main representative category is also the academic context (17.91\%), followed this time by other profiles $(9.95 \%)$ and incomplete protocol $(7.46 \%)$, with zero study based on architects' feedback.

Therefore, the three major axes of research development are: development of technologies such as new interfaces or methods; experiments in a controlled academic context tested on architecture students; experiments on other profiles such as end-users.

In order to clearly visualize the trend of the samples generally used in the last five years of research, we exclusively look at the participant profiles merging the prototype and experiment categories (Figure 4). The graph illustrates that researchers rely more on the academic context with students when testing new or existing immersive technologies. It also highlights the low rate of feedback from professional architects compared to other categories.

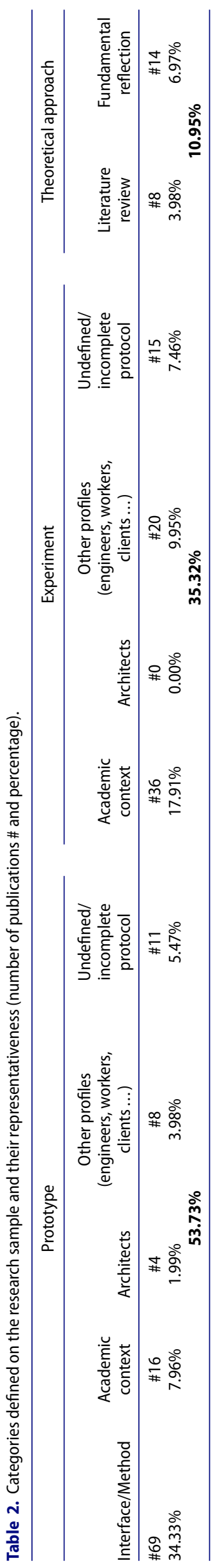




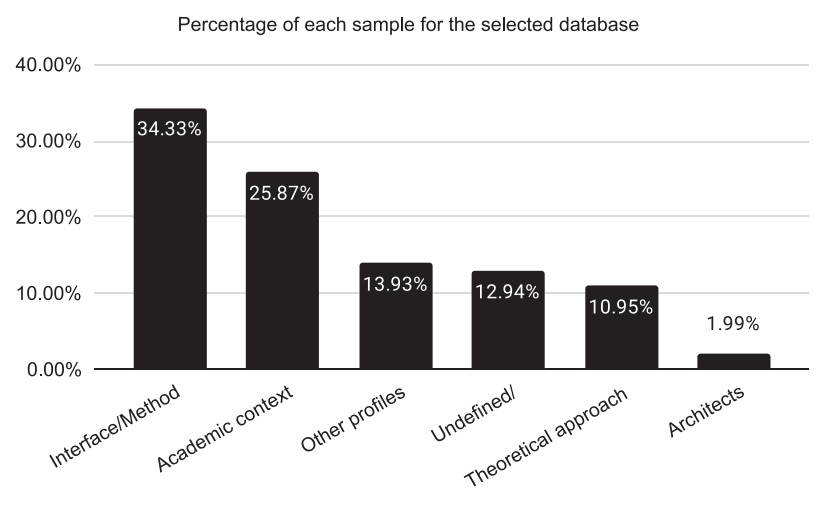

Figure 4. Representativeness of research sample, prototype and experiment categories merged.

Less than $2 \%$ of the 201 selected studies for the architects category means that only four papers reports architects' feedback. As our research interest is focused on immersive technology in the professional practice, we summarize in Table 3 the four related researches identified in our selection.

These four studies report the development of a tool improving the communication between the architects themselves or with other profiles such as the engineers or the clients.

The following section discusses considerations that may affect the results of this systematic literature review and identifies existing gaps in the current literature.

\section{Discussion}

The results of our review put light on the current gap in XR research. Researches on the $A E C$ sector are solving issues outside the scope of architects and are generally focusing on a specific immersive technology. For example, a literature review focusing on MR technology in the AEC industry is based on papers published in journals until 2018 (Cheng, Chen, and Chen 2020). However, of the 32 journals in the database, there is no journal devoted to the architectural field. Another study conducted in 2008 (Greenwood et al. 2008), analyses the use of virtual reality within the building industries in four countries (China, Sweden, the U.K. and the U.S.). The aims of the research were to assess VR usage and its benefits within the building industries of these countries and to identify perceived barriers to VR usage and ways of overcoming them. Authors have specifically selected large building companies and no architects have been interviewed during the process. These observations point to the need for a state-of-the-art review focusing on XR technology applications in the architecture field to reflect the current status of XR implementation.
Through a systematic literature review, the paper, therefore, provides a thorough analysis of contemporary publications related to immersive technologies in architecture, VR, AR, MR considered, and illustrates the current research trends over the past five years.

The release of more affordable and accurate devices between 2013 and 2015 corresponds with the increase of publications few years later as shown in Figure 3. This trend is also observed in a recent study focusing on CAADRIA publications over the past 25 years (Herr 2020).

Another study (Cerovsek and Martens 2020) analysing CAADRIA publications shows that immersive environments is one of the most studied subjects among other digital topics, mainly through the three keywords 'virtual reality', 'augmented reality', 'mixed reality'. At the same time, the low number of publications in journals and in particular in Architectural Science Review shows the importance of addressing this field of research in depth and therefore publishing accurate results in journals.

These two recent CAADRIA publications (Cerovsek and Martens 2020; Herr 2020) focus on keywords, highlighting the most frequently discussed topics. The particularity of our research is to look at the context of research. By this specific focus among recent publications about XR technologies, we highlight the high percentage of prototypes, and moreover the high percentage of the interface/method category, compared to experiments. These trends show that most of the publications relate on new developments, sometimes without further questioning the already existing tools and the relevance of their application.

Therefore, while some researchers consider XR technology to be widely adopted (Huang, White, and Burry 2018), few studies actually address the adoption of these new technologies by architects or even present the current rate of implementation in the professional practice. This trend seems in fact not to be evolving over the years as Moloney already noticed this distribution in 2009. He stated that many of the prototypes and case studies have been developed as university research projects that demonstrate technical feasibility, with scant regard for how the technology may be used and integrated with existing design practice (Moloney 2009). This trend could be reinforced by the number of publications classified as undefined/incomplete protocols or experiments. In most cases, it can be assumed that the profiles of the participants would have been classified in the academic context category relying on students.

This review also enables the identification of studies raising similar concerns but led in an academic context. Two studies question the relevance and consequences of the implementation of immersive technologies in architectural education. The first one conducted by Markusiewicz and Słyk (2015) presents

Table 3. Description of the research about XR technologies collecting architects' feedback.

\begin{tabular}{|c|c|c|c|}
\hline Reference & Country of research & Category & Research goal \\
\hline Coroado et al. (2015) & Portugal & Prototype & $\begin{array}{l}\text { Improve communication across different specialities enhancing project } \\
\text { decision process with a new VR tool }\end{array}$ \\
\hline Figen Gul and Halici (2016) & Turkey & Prototype & $\begin{array}{l}\text { Understand the interaction and collaborative communication of designers } \\
\text { using a mobile AR platform }\end{array}$ \\
\hline Costa et al. (2017) & Portugal & Prototype & $\begin{array}{l}\text { Enrich the interaction of designers with physical scale models of buildings } \\
\text { through an AR app }\end{array}$ \\
\hline Erzetic et al. (2019) & Australia & Prototype & $\begin{array}{l}\text { Enhance communication between the client and the designer through an } \\
\text { effective mobile AR interface }\end{array}$ \\
\hline
\end{tabular}


the process of implementing AR technology in architectural education. In the same way, the other research reports the role of VR as a new game changer in computational design education (Sorguç et al. 2017). Researchers study how the immersion in architectural design studio affects spatial perception through the design process, how it will improve students' spatial understanding of 3D volumes, and how it will enhance their imagination, enrich their creativity and promote their ability to experience their design's sensations. These questions have to be raised in a professional context in order to compare the results.

We can also point to a few studies with an approach that can possibly be associated with the limited means of small architectural offices. Nunes de Vasconcelos et al. (2018) as well as Dokonal and his colleagues (Dokonal, Knight, and Dengg 2016; Dokonal and Medeiros 2019) investigate the potential use of low-cost virtual reality devices in architectural education. Dokonal and his colleagues have indeed highlighted in previous studies that the major part of architects are working in small structure with low means (Dokonal, Knight, and Dengg 2016; Dokonal and Medeiros 2019) but led the experiments in an academic context with students without questioning the transposition of the results in a professional context.

If the academic context is an accessible environment offering interesting results based on students practice, even raising similar research questions between this context and a professional application, results should be reconsidered for each context. Indeed, this distinction is confirmed by a recent study comparing the integration of creative instruments employed for a digital design process in professional architectural practice vs educational environment (Rogers and Schnabel 2018). It emphasizes the relevance of using software in one context and not in the other (459):

The expert evaluations conclude that all design instruments and methodologies implemented within the digital design ecology work together well for educational purposes. Within the professional prac-

tice, however, the various tools could be implemented seamlessly; whereas some of them would not suit the industry from a time-cost perspective.

Finally, it is worth noting that this review is based on contemporary publications. Our review shows that a large part of studies is focusing on the development of interfaces, workflow or methods and moreover in the year 2019. Authors describe the development of the process itself without testing the results in a practical environment. This recent significant increase in the technology development could potentially be accompanied by a second phase of research, therefore concomitant with the writing of our paper. An example is the research conducted by Camacho and his colleagues in 2019 in which they announce a second phase to test the prototype (Camacho et al. 2019).

At the present time, our systematic review points an important development of technologies without questioning the real needs of architects to fit their day-to-day practice. As recently highlighted by Herr (2020), 'research field tends to be driven by technical innovation as well as a playful and speculative engagement with technology' (575) while some deeper considerations need to be taken into account in order to address more global challenges.

This literature review does not mean that the adoption phase of immersive technologies in architectural offices has not started yet, but it highlights that professional architects are finally not sufficiently integrated into the core of XR research in architecture.

While our results show that studies seem to agree on the significant potential improvement of immersive technologies as well as on their potential in the academic context, the transposition in the professional architectural practice and moreover, in small offices, is still to be demonstrated.

\section{Conclusion and future research}

Looking at research in the field of digital architecture, most of the research examining the prevalence of immersive technology within the architectural practice dates to twenty years ago and focused on a specific immersive technology (Bertol 1996; Frost and Warren 2000).

This contribution reviews 201 papers on VR, AR or MR technologies in the architectural field and classifies them into three main categories: prototype, experiment or theoretical approach, and sub-categories defined on the sample of participants.

Despite the increasing attention for immersive technology in education, few studies have been conducted on the current state of immersive technology research dedicated to professional architects to our knowledge.

Studies addressing XR evolution in the dataset we have defined are mainly focused on the development of tools and not on understanding the phenomena of adoption and the apprehensions that are formulated. The coexistence of these two scientific approaches is however essential if we aim to avoid the development of technologies not very appropriate and therefore not very sustainable.

There is a necessity to consider more carefully the context in which studies are conducted in order to develop tools truly dedicated to the practices of professional architects. Generally speaking, approaches based on the real needs of stakeholders ensure the development of models, methods, and even tools that respect daily practices while at the same time developing dimensions that can be improved. This approach thus enables researchers, and in particular software developers, to be more relevant in the development of a solution and, consequently, to see the results of their work being adopted more quickly and naturally by the actors concerned. The goal of this review is to promote a more conscientious and critical view on the evolution of immersive technologies within the architectural theory and practice.

Considering this literature review, the next phase of the research gathers professional architects in order to test and discuss the potential implementation of XR technologies in architectural offices of small size. Previous research has indeed shown that offices of smaller size represent the largest part of the market. Indeed, a European survey studying the architectural profession in Europe in 2018 reveals that $99 \%$ of offices are composed of less than ten people, and $71 \%$ of only one person (Architects' Council of Europe 2019). The study also showed that the number of medium-sized offices is continuously decreasing in favour of smaller structures. The goal of this study is a first step to question the effective practice of the largest part of the architecture market generally left aside and facing the digital transformation. 


\section{Disclosure statement}

No potential conflict of interest was reported by the author(s).

\section{Funding}

This work was supported by Université de Liège and Wallonie-Bruxelles International.

\section{ORCID}

Adeline Stals (D) http://orcid.org/0000-0001-9683-6395

\section{References}

Abdelhameed, Wael A. 2013. "Virtual Reality Use in Architectural Design Studios: A Case of Studying Structure and Construction." Procedia Computer Science 25: 220-230. doi:10.1016/j.procs.2013.11.027.

Architects' Council of Europe. 2019. "La Profession d'architecte En Europe 2018: Une Étude Du Secteur." Study by Mirza \& Nacey Research.

Bell, Lee. 2016. "Google Cardboard Launches in UK for £15." www.wired.co. uk.

Bertol, Daniel. 1996. Designing Digital Space: An Architect's Guide to Virtual Reality. New York: Wiley. doi:10.1016/s0010-4485(97)00065-1.

Billinghurst, Mark, and Anders Henrysson. 2009. "Mobile Architectural Augmented Reality." In Mixed Reality in Architecture, Design and Construction, edited by X. Wang and M. A. Schnabel, 93-104. Dordrecht: Springer.

Boell, S. K., and D. Cecez-Kecmanovic. 2015. "On Being 'Systematic' in Literature Reviews." In Formulating Research Methods for Information Systems, edited by L. P. Willcocks, C. Sauer, and M. C. Lacity. London: Palgrave Macmillan. https://link.springer.com/chapter/10.1057/9781137509888_3.

Bourbonnais, Sébastien. 2014. "Sensibilités Technologiques: Expérimentations et Explorations En Architecture Numérique 1987-2010." Thesis, Université de Laval Québec et Université de Paris-est.

Caldas, Luisa, and Mohammad Keshavarzi. 2019. "Design Immersion and Virtual Presence." Technology Architecture + Design 3 (2): 249-251. doi:10.1080/24751448.2019.1640544.

Camacho, Daniel, Tiara Dobbs, Alessandra Fabbri, Nicole Gardner, M. Hank Haeusler, and Yannis Zavoleas. 2019. "Hands on Design Thinking - Integrating Haptic Interaction and Feedback in Virtual Environments for Enhanced Immersive Experiences in Design Practice." In Intelligent \& Informed-CAADRIA Vol. 1, 563-572. doi:10.15358/9783800660742.

Carpo, Mario. 2017. The Second Digital Turn - Design Beyond Intelligence. Writing Architecture. Cambridge: MIT Press.

Cerovsek, Tomo, and Bob Martens. 2020. "The Evolution of CAADRA Conferences. A Bibliometric Approach" RE: Anthropocene, 25th CAADRIA, Bangkok, Vol. 1, 325-334.

Cheng, Jack C. P., Keyu Chen, and Weiwei Chen. 2020. "State-of-theArt Review on Mixed Reality Applications in the AECO Industry." Journal of Construction Engineering and Management 146 (2): 1-12. doi:10.1061/(ASCE)CO.1943-7862.0001749.

Coroado, Luís, Tiago Pedro, Jorge D’Alpuim, Sara Eloy, and Miguel Sales Dias. 2015. "VIARMODES - Visualization and Interaction in Immersive Virtual Reality for the Architectural Design Process." ECAADE 33 Virtual Reality-Evaluation, Vienna, Vol. 1, 125-134.

Costa, Fábio, Sara Eloy, Miguel Sales Dias, and Mariana Lopes. 2017. "ARch4models: A Tool to Augment Physical Scale Models." Design Tools - Theory - ECAADe, Rome, Vol. 1, 711-718.

Dokonal, Wolfgang, Mike Knight, and Ernst Dengg. 2016. "VR or Not VR - an EeZee Question?" In SIGraDi, 831-837. doi:10.5151/despro-sigradi2016777.

Dokonal, Wolfgang, and Marina Lima Medeiros. 2019. "I Want To Ride My Bicycle - I Want To Ride My Bike Using Low Cost Interfaces for Virtual Reality." ECAADe 37 / SIGraDi 23, Vol. 2, 465-472. http://papers.cumincad.org/ cgi-bin/works/paper/ecaadesigradi2019_309.

Dorta, Tomás, Stéphane Safin, Sana Boudhraâ, and Emmanuel Beaudry Marchand. 2019. "Co-Designing in Social VR." Intelligent and Informed CAADRIA, Wellington, Vol. 2, 141-150.

Erzetic, Catherine, Tiara Dobbs, Alessandra Fabbri, Nicole Gardner, M. Hank Haeusler, and Yannis Zavoleas. 2019. "Enhancing User-Engagement in the
Design Process Through Augmented Reality Applications." Simulation VIRTUAL AND AUGMENTED REALITY 1, Perth, Vol. 2, 423-432.

Farinea, Chiara, Areti Markopoulou, and Aldo Sollazzo. 2017. "Merging the Physical and Digital Layer of Public Space - the PobleJoc Installation Case Study." Virtual and Augmented Reality - ECAADe 35, Rome, Vol. 2, 725-730.

Figen Gul, Leman, and Süheyla Muge Halici. 2016. "Collaborative Design with Mobile Augmented Reality." Collaboration and Participation - Proceedings of the 34th ECAADe Conference, Oulu, Vol. 1, 493-500.

Frost, Peter, and Peter Warren. 2000. "Virtual Reality Used in a Collaborative Architectural Design Process." In IEEE International Conference on Information Visualization, 568-573. London: IEEE Comput. Soc. doi:10.1109/iv.2000.859814.

Greenwood, David, Margaret Horne, Emine Mine Thompson, Carl Martin Allwood, Claes Wernemyr, and Börje Westerdahl. 2008. "Strategic Perspectives on the Use of Virtual Reality Within the Building Industries of Four Countries." Architectural Engineering and Design Management 4 (2): 85-98. doi:10.3763/aedm.2008.0076.

Hanaoka, Ikuya, Seigo Tanaka, Alric Lee, and Kensuke Hotta. 2018. "Sight Depth Illusion with Perforated Plane - Evaluate in Mixed Reality with Head Mounted Display." Learning, Adapting and Prototyping, Proceedings of the 23rd International Conference of CAADRIA, Beijing, Vol. 1, edited by S. Alhadidi, T. Fukuda, W. Huang, P. Janssen, and K. Crolla, 411-420.

Heim, Michael R. 2017. "Virtual Reality Wave." In Boundaries of Self and Reality Online: Implications of Digitally Constructed Realities, edited by J. Gackenbach and J. Bown, 261-277. London: Academic Press.

Herr, Christiane M. 2020. "CAADRIA at Age 25: Mapping Our Past, Present, and Future." RE: Anthropocene, 25th CAADRIA, Bangkok, Vol. 2,567-576.

Huang, Xiaoran, Marcus White, and Mark Burry. 2018. "Design Globally, Immerse Locally." Learning, Adapting and Prototyping - CAADRIA, Beijing, Vol. 1, edited by T. Fukuda, W. Huang, P. Janssen, K. Crolla, and S. Alhadidi, 473-482.

Jeelani, Idris, Kevin Han, and Alex Albert. 2017. "Development of Immersive Personalized Training Environment for Construction Workers." Congress on Computing in Civil Engineering, 407-415. doi:10.1061/9780784480830. 050 .

Krakhofer, Stefan, and Martin Kaftan. 2015. "Augmented Reality Design Decision Support Engine for the Early Building Design Stage." Emerging Experiences in the Past, Present and Future of Digital Architecture - CAADRIA, Daegu, 231-240.

Kwiecinski, Krystian, Jacek Markusiewicz, and Agata Pasternak. 2017. "Participatory Design Supported with Design System and Augmented Reality." Shock - Sharing of Computable Knowledge - Proceedings of the 34th ECAADe Conference, Oulu, Vol. 2, 745-754.

Lescop, Laurent, Bruno Suner, and De Nantes. 2018. "15 Years of Immersion - Evolution and Assessment of a Pedagogy." CAAD Education| Practices ECAADe 36, Vol. 1, 391-400, Lodz.

Markusiewicz, Jacek, and Jan Słyk. 2015. "From Shaping to Information Modeling in Architectural Education: Implementation of Augmented Reality Technology in Computer-Aided Modeling." Real Time - Proceedings of the 33rd ECAADe Conference, Vienna, Vol. 2, 83-90.

Milgram, Paul, and Fumio Kishino. 1994. "Taxonomy of Mixed Reality Visual Displays." IEICE Transactions on Information Systems 77 (12): 1321 1329.

Miltiadis, Constantinos. 2015. "Virtual Architecture in a Real-Time, Interactive, Augmented Reality Environment - Project Anywhere Andthe Potential OfArchitecture in the Age Ofthe Virtual." Virtual Reality - Experimental ECAADe 33, Vienna, Vol. 1, 61-68.

Moloney, Jules. 2009. "Temporal Context and Concurrent Evaluation: Enhancing Decision Making at the Early Stages of Architectural Design with Mixed Reality Technology." In Mixed Reality in Architecture, Design, And Construction, edited by X. Wang and M. A. Schnabel, 135153. Dordrecht: Springer.

Moloney, Jules, Anastasia Globa, Rui Wang, and Chin Koi Khoo. 2019. "Principles for the Application of Mixed Reality as Pre-Occupancy Evaluation Tools (P-OET) at the Early Design Stages." Architectural Science Review: 1-10. doi:10.1080/00038628.2019.1675138.

Moore, Frank, and Masoud Gheisari. 2019. "A Review of Virtual and Mixed Reality Applications in Construction Safety Literature." Safety 5 (3): 1-16. doi:10.3390/safety5030051. 
Nunes de Vasconcelos, Guilherme, Mateus de Sousa Van Stralen, Alexandre Menezes, and Fernando Murilo Gontijo Ramos. 2018. "Perceive to Learn to Perceive: An Experience with Virtual Reality Devices for Architecture Design Learning." No. 1993: 985-90. doi:10.5151/sigradi2018-1364.

O'Hare, Jamie, Elies Dekoninck, Mendy Mombeshora, Philippe Martens, Niccolò Becattini, and Jean-Francois Boujut. 2018. "Defining Requirements for an Augmented Reality System to Overcome the Challenges of Creating and Using Design Representations in Co-Design Sessions." CoDesign: 1-24. doi:10.1080/15710882.2018.1546319.

Park, Hyejin, David Stephen Panya, Hyungmo Goo, Teahoon Kim, and Jihyo Seo. 2015. "BIM-Based Virtual Reality and Human Behavior Simulation for Safety Design." Proceedings of the 36th eCAADe Conference - Volume 2, Lodz University of Technology, Lodz, Poland, 823-832.

Poustinchi, Ebrahim. 2018. "Subtractive Digital Fabrication with Actual Robot and Virtual Material Using a MARI Platform." International Journal of Architectural Computing 16 (4): 281-294. doi:10.1177/1478077118801594.

Rogers, Jessie, and Marc Aurel Schnabel. 2018. “Digital Design Ecology - An Analysis for an Intricate Framework of Architectural Design." Collaborative \& Participative Desgin - ECAADe 36, Lodz, Poland, Vol. 1, 459-468.

Schnabel, Marc Aurel. 2004. "Architectural Design in Virtual Environments Exploring Cognition and Communication in Immersive Virtual Environments." PhD thesis, University of Hong Kong. doi:10.1016/S0142-694X (97)85457-9.
Schnabel, Marc Aurel, Thomas Kvan, Ernst Kruijff, and Dirk Donath. 2001. "The First Virtual Environment Design Studio." 19th ECAADe Conference Proceedings, Helsinki, 394-400.

Sheldon, Aron, Tiara Dobbs, Alessandra Fabbri, Nicole Gardner, M. Hank Haeusler, Cristina Ramos, and Yannis Zavoleas. 2019. "Putting the AR in (Ar)chitecture." Intelligent and Informed - CAADRIA, Wellington.

Shi, Yangming, Jing Du, Sarel Lavy, and Dong Zhao. 2016. "A Multiuser Shared Virtual Environment for Facility Management." Procedia Engineering 145: 120-127. doi:10.1016/j.proeng.2016.04.029.

Snyder, Hannah. 2019. "Literature Review as a Research Methodology: An Overview and Guidelines." Journal of Business Research 104: 333-339. doi:10.1016/j.jbusres.2019.07.039.

Sorguç, Arzu Gönenç, Müge Kruşa Yemişcioğlu, Çağlar Fırat Özgenel, Mert Ozan Katipoğlu, and Ramin Rasulzade. 2017. "The Role of VR as a New Game Changer in Computational Design Education." ECAADe 35, VOI. 1, 401-408. http://papers.cumincad.org/data/works/att/ecaade2017_142. pdf.

Spaeth, A Benjamin, and Ramez Khali. 2018. "The Place of VR Technologies in UK Architectural Practice." Architectural Engineering and Design Management 14 (6): 470-487. doi:10.1080/17452007.2018.1502654.

Wang, Xiangyu, and Phillip S. Dunston. 2007. "Design, Strategies, and Issues Towards an Augmented Reality-Based Construction Training Platform." Electronic Journal of Information Technology in Construction 12: 363-380. 